\title{
Forced Vibrations of the Hopper of Fertilizer Applying Machine
}

\author{
Sayakhat NUKESHEV*, Valentin SLAVOV**, Dimitar KARAIVANOV**, \\ Aigul BALABEKOVA*, Ziyada ZHAKSYLYKOVA* \\ *Kazakh Agro Technical University named after S. Seifullin, Pobedy 62, 010011 Astana, Kazakhstan, \\ E-mail: nuk.sayakhat@gmail.com \\ **University of Chemical Technology and Metallurgy, 8 Kl. Ohridski Blvd. Sofia 1756, Bulgaria \\ crossref $\mathrm{http}: / / \mathrm{dx}$. doi.org/10.5755/j01.mech.24.6.22464
}

\section{Introduction}

To date, soil application of mineral fertilizers is one of the key factors in increasing the yield [1-3]. This leads to higher requirements to the mineral fertilizers, which are aimed at obtaining the maximum number of agricultural products, as well as compliance with environmental safety $[4,5]$. For soils prone to erosion, almost the only way to carry out intensive farming will be tilter-free plowing. This practice significantly reduces the risk of soil erosion, but there are problems with other agricultural technicians, as it is fertilizing, sowing material, etc. historically optimized for widely used dump plowing and in the case of application of soilless - lead to unproductive fertilizer consumption and sowing material.

One of the advanced technology in modern agriculture is the intra soil differentiated (precisely) fertilization - the introduction of exact amounts of fertilizer directly to the roots of plants (or in the furrow at seeding). A common feature of the machines for this type of fertilizer is that the fertilizer is fed from a mine hopper to multiple tines (working bodies), which put it into the soil. Studies have shown that to realize maximal effect of this method it will be necessary that all the tines uniformly feed the same amount of fertilizer. Improving the uniformity of fertilizing increases yields of cereals to $15-20 \%[6,7]$.

Among the existing systems for feeding (sowing) the material (fertilizer, seeds) in the soil most attention deserve the pneumatic ones, in which a flexible pipe takes the material to the working bodies [8-10]. The advantages of this apparatus are: the high frequency oscillations make to the seeding system resistant to external factors, created a major force capable to destroy any connection between the individual elements of the body [11-13]. The main problem with them is uniform submission of the material to any pipeline of the system. The basis of the solution of this problem is to design a dosing device which provides uniform delivery of fertilizer to all working bodies.

There are different designs of dosing devices for granular bulk material, and as a rule they do not provide a uniform density of the material flow in the whole section because it was not necessary in the cases for which they were designed [14-15].

The object of this study is a dosing device which is being developed by young scientists from Kazakhstan [16].

The purpose of this research is to build a dynamic model to describe the behaviour of a hopper under forced vibration. Since a dosing hopper operates under forced oscillations (vibration) to achieve a uniform distribution of the sown material, oscillations that take place in particular units and in the mechanisms cannot be neglected. This is why this work - a model describing the behaviour of a dynamic system, and a system in general, with vibrations at different frequencies - is needed. One of the goals set was to investigate unit performance at frequencies of vibration that are close to resonant frequency. If the system does not enter into resonance in any of operating modes that give a uniform fertilizer/seed application, dynamic system design will be assumed successful.

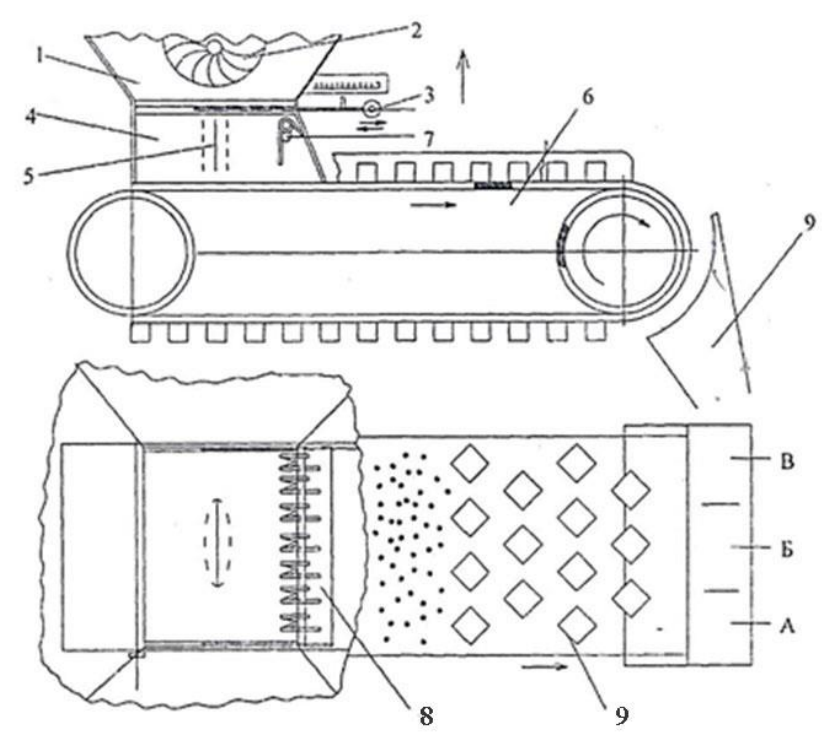

Fig. 1 Design of the dosing device

The design of the dosing device is shown in Fig. 1.

The material at the bottom of the hopper 1 is loosened by 2 and entering the chamber 4 , wherein the vibrating plate 5 provides to fill the entire cross section and uniformly distributes of the material on the conveyor belt 6 . An equalizer 7 and relief of the belt 8 contribute to improving the uniformity of the flow.

\section{Method}

A dynamic model of the dosing device is shown in Fig. 2.

Body 1 (hopper) is set to 4 elastic damping supports (shown on corners of lower part of hopper) - makes small-amplitude movements along the axes of the coordinate system $O_{1} x_{1} y_{1} z_{1}$ and small-amplitude rotations about the same axis. It has 6 degrees of freedom. Body 2 (rod) makes small-amplitude movements along the axis $\mathrm{O}_{2} x_{2}$ - 
translation relative to body 1 due to the prismatic connection between the two bodies.

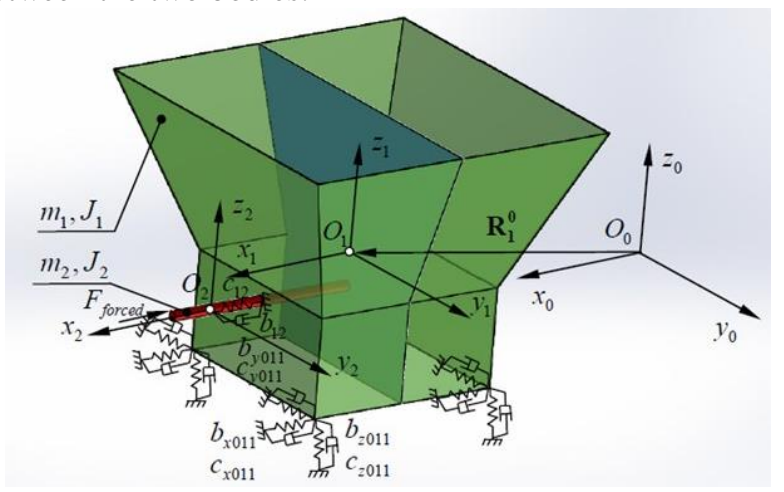

Fig. 2 Dynamic model of the dosing device

There is a linear-elastic damping element between body 1 and body 2 . Forced vibrations are excited by electromagnet, acting on the axis $O_{2 \times 2}$ on body 2 . The mechanical system is with 7 degrees of freedom.

\subsection{Kinematics}

To determine the kinetic and potential energy, energy of dissipation, and generalized forces, kinematics of the mechanical system must first be studied - positions of points (mass centers) and speed - angular of the bodies and linear of the points [14].

A vector of generalized coordinates that define the position of the mechanical system of bodies in space is:

$$
\mathbf{q}=\left[\begin{array}{lllllll}
x_{1} & y_{1} & z_{1} & \theta_{x 1} & \theta_{y 1} & \theta_{z 1} & x_{2}
\end{array}\right]^{\mathrm{T}} .
$$

Matrices of transformation through which the vectors are projected in reference coordinate system are:

- for body 1 :

$$
\mathbf{A}_{1}^{0}=\mathbf{A t}_{1}^{0} \cdot \mathbf{A u}_{1}^{0}
$$

where:

$$
\begin{aligned}
\mathbf{A t}_{\mathbf{1}}^{\mathbf{0}} & =\left[\begin{array}{cccc}
1 & 0 & 0 & l_{x 01}+x_{1} \\
0 & 1 & 0 & l_{y 01}+y_{1} \\
0 & 0 & 1 & l_{z 01}+z_{1} \\
0 & 0 & 0 & 1
\end{array}\right] \quad \mathbf{R}_{\mathbf{1}}^{\mathbf{0}}=\left[\begin{array}{c}
l_{x 01} \\
l_{y 01} \\
l_{z 01} \\
1
\end{array}\right], \\
\mathbf{A} \mathbf{u}_{\mathbf{1}}^{\mathbf{0}} & =\left[\begin{array}{cccc}
1 & -\theta_{z 1} & \theta_{y 1} & 0 \\
\theta_{z 1} & 1 & -\theta_{x 1} & 0 \\
-\theta_{y 1} & \theta_{x 1} & 1 & 0 \\
0 & 0 & 0 & 1
\end{array}\right] .
\end{aligned}
$$

- for body 2 :

$$
\mathbf{A}_{2}^{0}=\mathbf{A}_{1}^{0} \cdot \mathbf{A}_{2}^{1},
$$

where:

$$
\mathbf{A}_{2}^{\mathbf{1}}=\left[\begin{array}{cccc}
1 & 0 & 0 & l_{x 12}+x_{2} \\
0 & 1 & 0 & l_{y 12} \\
0 & 0 & 1 & l_{z 12} \\
0 & 0 & 0 & 1
\end{array}\right] .
$$

Vectors of the position of the mass centers of the bodies projected in the reference coordinate system are:

- for body 1 :

$$
\mathbf{R}_{\mathbf{C} \mathbf{1}}^{\mathbf{0}}=\mathbf{A}_{\mathbf{1}}^{\mathbf{0}} \cdot \mathbf{r}_{\mathbf{C} 1} ; \quad \mathbf{r}_{\mathbf{C} \mathbf{1}}=\left[\begin{array}{llll}
l_{x C 1} & l_{y C 1} & l_{z C 1} & 1
\end{array}\right]^{\mathrm{T}} ;
$$

- for body 2 :

$$
\mathbf{R}_{\mathbf{C} 2}^{\mathbf{0}}=\mathbf{A}_{\mathbf{2}}^{\mathbf{0}} \cdot \mathbf{r}_{\mathbf{C} 2} ; \quad \mathbf{r}_{\mathbf{C} 2}=\left[\begin{array}{llll}
l_{x C 2} & l_{y C 2} & l_{z C 2} & 1
\end{array}\right]^{\mathrm{T}} .
$$

Vectors of absolute linear velocity of the mass centers projected in reference coordinate system are:

- for body 1 :

$$
\mathbf{V}_{\mathbf{C} 1}^{\mathbf{0}}=\frac{\mathrm{d} \mathbf{R}_{\mathbf{C} 1}^{\mathbf{0}}}{\mathrm{dt}}=\left[\sum_{\mathrm{k}=1}^{6}\left(\frac{\partial \mathbf{A}_{1}^{\mathbf{0}}}{\partial \mathrm{q}_{\mathrm{k}}}\right) \cdot \dot{\mathrm{q}}_{\mathrm{k}}\right] \cdot \mathbf{r}_{\mathrm{C} 1}=\left(\frac{\partial \mathbf{A}_{1}^{\mathbf{0}}}{\partial x_{1}} \cdot \dot{x}_{1}+\frac{\partial \mathbf{A}_{1}^{\mathbf{0}}}{\partial y_{1}} \cdot \dot{y}_{1}+\frac{\partial \mathbf{A}_{1}^{\mathbf{0}}}{\partial z_{1}} \cdot \dot{z}_{1}+\frac{\partial \mathbf{A}_{1}^{\mathbf{0}}}{\partial \Phi_{1}} \cdot \dot{\Phi}_{1}+\frac{\partial \mathbf{A}_{1}^{\mathbf{0}}}{\partial \Phi_{2}} \cdot \dot{\Phi}_{2}+\frac{\partial \mathbf{A}_{1}^{\mathbf{0}}}{\partial \Phi_{3}} \cdot \dot{\Phi}_{3}\right) \cdot \mathbf{r}_{\mathbf{C} 1} ;
$$

- for body 2 :

$$
\mathbf{V}_{\mathbf{C} 2}^{\mathbf{0}}=\frac{\mathrm{d} \mathbf{R}_{\mathbf{C} 2}^{\mathbf{0}}}{\mathrm{dt}}=\left[\sum_{\mathrm{k}=1}^{6}\left(\frac{\partial \mathbf{A}_{2}^{\mathbf{0}}}{\partial \mathrm{q}_{\mathrm{k}}}\right) \cdot \dot{\mathrm{q}}_{\mathrm{k}}\right] \cdot \mathbf{r}_{\mathrm{C} 2}=\left(\frac{\partial \mathbf{A}_{2}^{\mathbf{0}}}{\partial x_{1}} \cdot \dot{x}_{1}+\frac{\partial \mathbf{A}_{2}^{\mathbf{0}}}{\partial y_{1}} \cdot \dot{y}_{1}+\frac{\partial \mathbf{A}_{2}^{\mathbf{0}}}{\partial z_{1}} \cdot \dot{z}_{1}+\frac{\partial \mathbf{A}_{2}^{\mathbf{0}}}{\partial \Phi_{1}} \cdot \dot{\Phi}_{1}+\frac{\partial \mathbf{A}_{2}^{\mathbf{0}}}{\partial \Phi_{2}} \cdot \dot{\Phi}_{2}+\frac{\partial \mathbf{A}_{2}^{\mathbf{0}}}{\partial \Phi_{3}} \cdot \dot{\Phi}_{3}+\frac{\partial \mathbf{A}_{2}^{\mathbf{0}}}{\partial x_{2}} \cdot \dot{x}_{2}\right) \cdot \mathbf{r}_{\mathrm{C} 2}
$$

Vectors of absolute angular velocity of bodies pro- $\quad$ - for body 1 : jected in local coordinate systems (they are necessary to calculate the kinetic energy of the mechanical system) are:

$$
\mathbf{\Omega}_{1}^{\mathbf{1}}=\left[\begin{array}{c}
\Omega_{1}^{1} \\
\Omega_{2}^{1} \\
\Omega_{3}^{1} \\
0
\end{array}\right]=\mathbf{A}_{\mathbf{1}}^{\mathbf{\Omega 1}} \cdot \dot{\boldsymbol{\Theta}}=\left[\begin{array}{c}
\dot{\Phi}_{1} \cdot \cos \Phi_{2} \cdot \cos \Phi_{3}+\dot{\Phi}_{2} \cdot \sin \Phi_{3} \\
\dot{\Phi}_{2} \cdot \cos \Phi_{3}-\dot{\Phi}_{1} \cdot \cos \Phi_{2} \cdot \sin \Phi_{3} \\
\dot{\Phi}_{3}+\dot{\Phi}_{1} \cdot \sin \Phi_{2} \\
0
\end{array}\right],
$$

where: 
$\mathbf{A}_{1}^{\mathbf{\Omega}}=\left[\begin{array}{cccc}\cos \Phi_{2} \cdot \cos \Phi_{3} & \sin \Phi_{3} & 0 & 0 \\ -\cos \Phi_{2} \cdot \sin \Phi_{3} & \cos \Phi_{3} & 0 & 0 \\ \sin \Phi_{2} & 0 & 1 & 0 \\ 0 & 0 & 0 & 1\end{array}\right] ;$

$\dot{\boldsymbol{\Theta}}=\left[\begin{array}{c}\dot{\Phi}_{1} \\ \dot{\Phi}_{2} \\ \dot{\Phi}_{3} \\ 0\end{array}\right]$

- for body 2 :

$$
\mathbf{\Omega}_{2}^{2}=\mathbf{\Omega}_{1}^{1}
$$

\subsection{Dynamics}

The kinetic energy of the mechanical system, the potential energy of deformation of the elastic elements are calculated as well as from the weights of the bodies the energy of dispersion of the damping elements was calculated [14]. From kinetic energy through differentiation in generalized speeds are obtained matrix of mass-inertial properties of the mechanical system. From potential energy by differentiating a generalized coordinate are obtained matrix of elastic properties of the mechanical system. From the energy of dispersion by differentiation in generalized speeds are obtained matrix of the damping properties of the mechanical system. Generalized forces are calculated taking into account the forces that excite vibrations and their applied points.

Using the Lagrange's equation of $2^{\text {nd }}$ kind, a system differential equations is compiled which describes the forced small-amplitude oscillations of the mechanical system.

\subsection{Kinetic energy}

The kinetic energy of the system is the sum of kinetic energies of the two bodies:

$$
T=\frac{1}{2} \sum_{i=1}^{2}\left(\mathbf{m}_{\mathbf{R R}}^{\mathrm{i}} \cdot \mathbf{V}_{\mathbf{C i}}^{\mathbf{0} \mathrm{T}} \cdot \mathbf{V}_{\mathbf{C i}}^{\mathbf{0}}+\mathbf{\Omega}_{\mathbf{i}}^{\mathrm{iT}} \cdot \mathbf{J}_{\mathbf{\Theta \Theta}}^{\mathrm{i}} \cdot \mathbf{\Omega}_{\mathbf{i}}^{\mathrm{i}}\right)
$$

where: $\boldsymbol{\Omega}_{\mathbf{i}}^{\mathbf{i}}$ is vector of absolute angular velocity of the respective body, projected in the local coordinate system; $\mathbf{V}_{\mathbf{C i}}^{\mathbf{0}}$ is vector of absolute velocity of mass center of the respective body, projected in the reference coordinate system;

$$
\begin{aligned}
\mathbf{m}_{\mathbf{R R}}^{\mathbf{i}} & =\left[\begin{array}{ccc}
m_{i} & 0 & 0 \\
0 & m_{i} & 0 \\
0 & 0 & m_{i}
\end{array}\right] ; m_{i} \text { is mass of the respective body; } \\
\mathbf{J}_{\Theta \Theta}^{\mathbf{i}} & =\left[\begin{array}{ccc}
\mathbf{J}_{\mathrm{xx}} & -\mathbf{J}_{\mathrm{xy}} & -\mathbf{J}_{\mathrm{xz}} \\
-\mathbf{J}_{\mathrm{xy}} & \mathbf{J}_{\mathrm{yy}} & -\mathbf{J}_{\mathrm{yz}} \\
-\mathbf{J}_{\mathrm{xz}} & -\mathbf{J}_{\mathrm{yz}} & \mathbf{J}_{z z}
\end{array}\right] \text { is inertia tensor of the relevant }
\end{aligned}
$$

body in the local coordinate system.

Matrix of mass and inertial properties:

$$
\mathbf{M}=\left[m_{i j}\right] ; \quad m_{i j}=\frac{\partial T}{\partial \dot{\mathrm{q}} \cdot \partial \dot{\mathrm{q}}}
$$

\subsection{Potential energy}

The potential energy of the mechanical system is the sum of the potential energies of deformation of any flexible elements and the weights of the two bodies:

$$
\Pi=\sum_{k=1}^{5} \Pi_{k}+\sum_{i=1}^{2} \Pi_{G i}
$$

where: $\Pi_{k}=\frac{1}{2} \cdot \mathbf{c}^{P} \cdot\left(\delta \mathbf{r}_{\mathbf{N}}^{\mathrm{ij}}\right)^{2}, \mathbf{c}^{P}$ is coefficient of elasticity; $\delta \mathbf{r}_{\mathrm{N}}^{\mathrm{ij}}$ is deformation of an elastic element;

$\Pi_{G i}=-m_{i} \cdot \mathbf{g}^{\mathrm{T}} \cdot \mathbf{R}_{\mathbf{C i}}^{\mathbf{0}}, \mathbf{g}^{\mathrm{T}}=\left[\begin{array}{llll}0 & 0 & \mathrm{~g} & 0\end{array}\right]^{\mathrm{T}}$ is vector that defines the gravitational acceleration at the reference coordinate system; $\mathbf{R}_{\mathbf{C i}}^{\mathbf{0}}$ is vector of a mass center position of the body $\mathrm{i}$, defined in the reference coordinate system.

A matrix of elastic properties is:

$$
\mathbf{C}=\left[c_{i j}\right] ; \quad c_{i j}=\frac{\partial \Pi}{\partial q \cdot \partial \mathbf{q}} .
$$

\subsection{Energy of dissipation}

The energy dissipation of the mechanical system is the sum of the energies of dissipation of all damping elements:

$$
R=\frac{1}{2} \sum_{i=1}^{5} \mathbf{b}^{P} \cdot\left(\delta \mathbf{V}_{\mathbf{N}}^{\mathrm{ij}}\right)^{2}
$$

where: $\mathbf{b}^{P}$ is damping coefficient; $\delta \mathbf{V}_{\mathbf{N}}^{\mathrm{ij}}$ is speed of deformation of the elastic element.

A matrix of damping properties is:

$$
\mathbf{B}=\left[b_{i j}\right] ; \quad b_{i j}=\frac{\partial R}{\partial \dot{\mathbf{q}} \cdot \partial \dot{\mathbf{q}}} .
$$

Generalized forces.

A vector of generalized forces is:

$$
\mathbf{Q}=\left[\begin{array}{lllllll}
0 & 0 & 0 & 0 & 0 & 0 & Q_{F}
\end{array}\right]^{\mathrm{T}},
$$

where: $Q_{F}=F_{\text {forc }} . \sin (\omega . t)$.

\section{Results}

Differential equations describing the forced vibrations of the mechanical system.

A system of differential equations which describes the forced small-amplitude oscillations of the mechanical system is:

$$
\mathbf{M}_{7 \times 7} \cdot \ddot{\mathbf{q}}_{7 \times 1}+\mathbf{B}_{7 \times 7} \cdot \dot{\mathbf{q}}_{7 \times 1}+\mathbf{C}_{7 \times 7} \cdot \mathbf{q}_{7 \times 1}=\mathbf{Q}_{7 \times 1} .
$$


Solution of the differential equations.

Finding general solutions of the system (21) is related to the determination of the initial conditions of motion $q(0)$ and $\dot{q}(0)$, which depend on the movement of the system.

$$
\begin{aligned}
& q(t)=\sum_{r=1}^{7} \frac{2}{g_{r}^{2}+h_{r}^{2}}\left[\mathbf{G}_{r} \mathbf{M} \dot{q}(0)+\left(-\alpha_{r} \mathbf{G}_{r} \mathbf{M}+\beta_{r} \mathbf{H}_{r} \mathbf{M}+\mathbf{G}_{r} \mathbf{B}\right) q(0)\right] \cdot e^{-\alpha_{r} t} \cdot \cos \beta_{r} t+ \\
& +\sum_{r=1}^{7} \frac{2}{g_{r}^{2}+h_{r}^{2}}\left[\mathbf{H}_{r} \cdot \mathbf{M} \cdot \dot{q}(0)+\left(-\alpha_{r} \cdot \mathbf{H}_{r} \cdot \mathbf{M}-\beta_{r} \cdot \mathbf{G}_{r} \cdot \mathbf{M}+\mathbf{H}_{r} \cdot \mathbf{B}\right) \cdot q(0)\right] \cdot e^{-\alpha_{r} t} \cdot \sin \beta_{r} t+ \\
& +\operatorname{Re}\left\{\sum_{k=0}^{n} \sum_{r=1}^{7} \frac{2}{g_{r}^{2}+h_{r}^{2}} \frac{\alpha_{r} \cdot \mathbf{G}_{r}+\beta_{r} \cdot \mathbf{H}_{r}+i \cdot k \cdot \Omega \cdot \mathbf{G}_{r}}{\omega_{r}^{2}-k^{2} \cdot \Omega^{2}+i \cdot 2 \cdot k \cdot \sigma_{r} \cdot \omega_{r} \cdot \Omega} \mathbf{Q} \cdot e^{i k \Omega t}\right\} .
\end{aligned}
$$

where: $\mathbf{Q}$ is the vector of generalized external forces;

and other symbols are:

$$
\begin{aligned}
& g_{r}=-2 \alpha_{r}\left(\mathbf{V}_{r}^{\mathrm{T}} \cdot \mathbf{M} \cdot \mathbf{V}_{r}-\mathbf{W}_{r}^{\mathrm{T}} \cdot \mathbf{M} \cdot \mathbf{W}_{r}\right)-4 \beta_{r} \mathbf{V}_{r}^{\mathrm{T}} \cdot \mathbf{M} \cdot \mathbf{W}_{r}+\mathbf{V}_{r}^{\mathrm{T}} \cdot \mathbf{B} \cdot \mathbf{V}_{r}-\mathbf{W}_{r}^{\mathrm{T}} \cdot \mathbf{B} \cdot \mathbf{W}_{r} ; \\
& h_{r}=2 \beta_{r}\left(\mathbf{V}_{r}^{\mathrm{T}} \cdot \mathbf{M} \cdot \mathbf{V}_{r}-\mathbf{W}_{r}^{\mathrm{T}} \cdot \mathbf{M} \cdot \mathbf{W}_{r}\right)-4 \alpha_{r} \mathbf{V}_{r}^{\mathrm{T}} \cdot \mathbf{M} \cdot \mathbf{W}_{r}+2 \mathbf{V}_{r}^{\mathrm{T}} \cdot \mathbf{B} \cdot \mathbf{W}_{r} ; \\
& \mathbf{G}_{r}=g_{r} \mathbf{L}_{r}+h_{r} \mathbf{R}_{r} ; \mathbf{L}_{r}=\mathbf{V}_{r} \cdot \mathbf{V}_{r}^{\mathrm{T}}-\mathbf{W}_{r} \cdot \mathbf{W}_{r}^{\mathrm{T}} ; \mathbf{H}_{r}=h_{r} \mathbf{L}_{r}-g_{r} \mathbf{R}_{r} ; \mathbf{R}_{r}=\mathbf{V}_{r} \cdot \mathbf{W}_{r}^{\mathrm{T}}+\mathbf{W}_{r} \cdot \mathbf{V}_{r}^{\mathrm{T}} .
\end{aligned}
$$

As an example, a small model of a real fertilizer hopper is solved, developed in Kazakhstan Agro Technical University, Astana, Republic of Kazakhstan (Fig. 3).

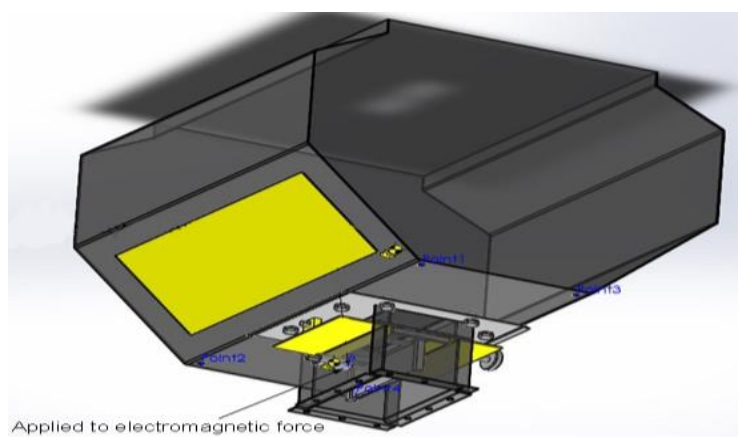

Fig. 3 Small model of a real fertilizer hopper 1 together with chamber 4 from Fig. 1

\section{Discussion}

As an example, for the calculation, there is considered a mechanical system with investigated coulter scatterers of the fertilizer and accepted real constructive parameters.

In Table 1, parameters of the small model of the fertilizer hopper are given as Input data (verification was performed on a reduced large-scale copy of a real hopper; calculations were made using data obtained with a scaled model to compare the results): masses; mass inertial mo-

\begin{tabular}{|c|c|c|c|c|c|c|c|c|c|c|}
\hline & \multicolumn{2}{|l|}{$m, \mathrm{~kg}$} & \multicolumn{2}{|l|}{$J_{x x}, \mathrm{~kg} \cdot \mathrm{m}^{2}$} & $J_{y y}, \mathrm{~kg} \cdot \mathrm{m}^{2}$ & \multicolumn{2}{|c|}{$J_{z z}, \mathrm{~kg} \cdot \mathrm{m}^{2}$} & $J_{x y}, \mathrm{~kg} \cdot \mathrm{m}^{2}$ & $J_{x z}, \mathrm{~kg} \cdot \mathrm{m}^{2}$ & $J_{y z}, \mathrm{~kg} \cdot \mathrm{m}^{2}$ \\
\hline Body 1 & \multicolumn{2}{|l|}{25.13} & \multicolumn{2}{|l|}{1.727} & 1.6575 & \multicolumn{2}{|l|}{1.7261} & 0 & 0 & 0 \\
\hline \multirow[t]{2}{*}{ Body 2} & \multicolumn{2}{|c|}{0.1068} & 0.000006 & & 0.000056 & \multicolumn{2}{|c|}{0.000056} & 0 & 0 & 0 \\
\hline & $c_{x}, \mathrm{~N} / \mathrm{m}$ & $c_{y}, \mathrm{~N} / \mathrm{m}$ & $c_{z}, \mathrm{~N} / \mathrm{m}$ & $b_{x}, \mathrm{~N} \cdot \mathrm{s} / \mathrm{m}$ & $b_{y}, \mathrm{~N} \cdot \mathrm{s} / \mathrm{m}$ & $b_{z}, \mathrm{~N} \cdot \mathrm{s} / \mathrm{m}$ & p. & $l_{k x}, \mathrm{~m}$ & $l_{k y}, \mathrm{~m}$ & $l_{k z}, \mathrm{~m}$ \\
\hline \multirow{4}{*}{ Body 1} & \multirow{4}{*}{$35 \cdot 10^{3}$} & \multirow{4}{*}{$35 \cdot 10^{3}$} & \multirow{4}{*}{$80 \cdot 10^{3}$} & \multirow{4}{*}{98} & \multirow{4}{*}{67} & \multirow{4}{*}{47} & 1 & 0.110 & 0.260 & -0.173 \\
\hline & & & & & & & 2 & 0.110 & -0.260 & -0.173 \\
\hline & & & & & & & 3 & -0.109 & 0.260 & -0.173 \\
\hline & & & & & & & 4 & -0.109 & -0.260 & -0.173 \\
\hline Body 2 & \multicolumn{10}{|l|}{$5 \cdot 10^{3}$} \\
\hline$F_{i x}, \mathrm{~N}$ & \multicolumn{2}{|c|}{\begin{tabular}{l|l|} 
& $F_{i y}, \mathrm{~N}$ \\
\end{tabular}} & $F_{i z}, \mathrm{~N}$ & $M_{i x}, \mathrm{~N} \cdot \mathrm{m}$ & $M_{i y}, \mathrm{~N} \cdot \mathrm{m}$ & \multicolumn{2}{|c|}{$M_{i z}, \mathrm{~N} \cdot \mathrm{m}$} & $l_{P x}, \mathrm{~m}$ & $l_{P y}, \mathrm{~m}$ & $l_{P z}, \mathrm{~m}$ \\
\hline $50 \sin (50 t)$ & \multicolumn{2}{|c|}{0} & 0 & 0 & 0 & \multicolumn{2}{|c|}{0} & 0.054 & 0 & -0.267 \\
\hline
\end{tabular}
ments, geometrical parameters, elastic and damping characteristics of elastic-damping elements and exciting forces. The reference coordinate system coincides with the coordinate system of body 1 .

Parameters of the small model of studied fertilizer hopper

Coordinates in $\mathrm{m}$ of the centre of coordinate system of body 2 are as follow:

$$
\begin{aligned}
& l_{x 2}^{1}=0.7383 ; \\
& l_{y 2}^{1}=0 ; \\
& l_{z 2}^{1}=0.2669 .
\end{aligned}
$$

Numerical and graphical results for free, damped and forced vibrations of the mechanical system (mentioned mechanical system shown on Fig. 2):

Natural frequencies, Hz:

$\left[\begin{array}{c}34.59 \\ 20.26 \\ 17.92 \\ 14.96 \\ 12.58 \\ 10.40 \\ 5.95\end{array}\right]$.

Natural forms (relative units): 


$$
\left[\begin{array}{ccccccc}
0 & 0 & 0 & 0 & 0.02 & 0 & -0.99 \\
0 & -0.09 & 0 & -0.99 & 0 & -0.01 & 0 \\
0.01 & 0 & -0.99 & 0 & -0.08 & 0 & 0.01 \\
0.28 & 0 & 0.01 & 0 & -0.96 & 0 & 0.01 \\
0 & 0,01 & 0 & -0.01 & 0 & -0.99 & 0 \\
0 & 0,6 & 0 & -0.8 & 0 & 0.07 & 0 \\
0.23 & 0 & 0 & 0 & 0,97 & 0 & 0.01
\end{array}\right]
$$

Fig. 4 shows 3D graphic of generalized coordinates, natural frequencies and natural forms of the mechanical system.

In Fig. 5 the diagrams of free damped vibrations of the system at all generalized coordinates are given. In Fig. 6 the diagrams of forced vibrations of the system at all generalized coordinates are given.
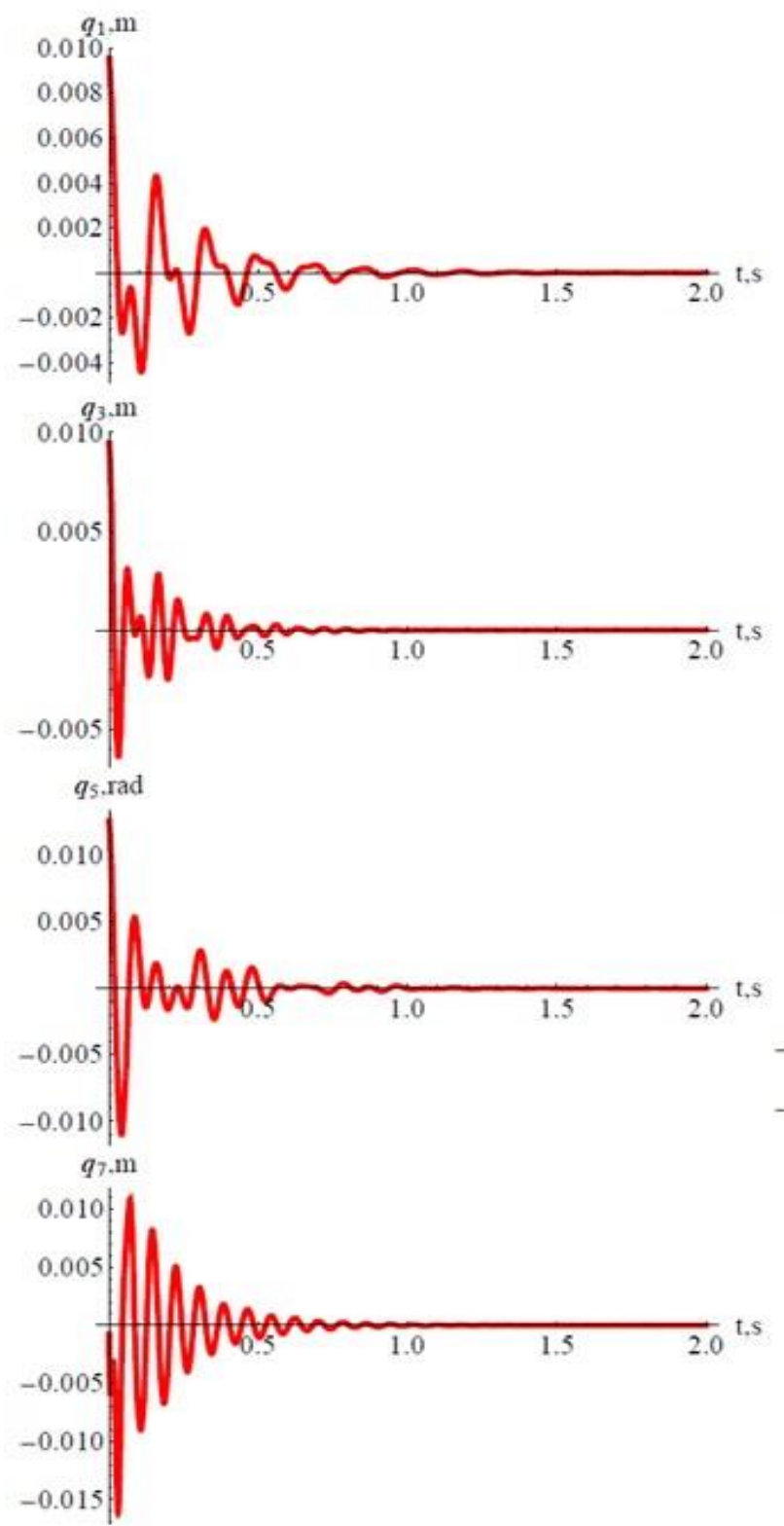

Fig. 5 Diagrams of free damped vibrations of the system at all generalized coordinates
In Fig. 7 the diagrams of amplitude-frequency characteristics of the system at all generalized coordinates are given.

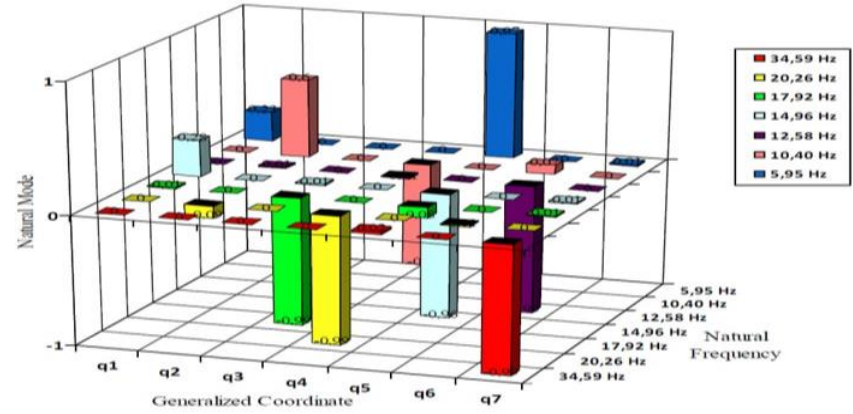

Fig. 4 Generalized coordinates, natural frequencies and natural forms of the mechanical system
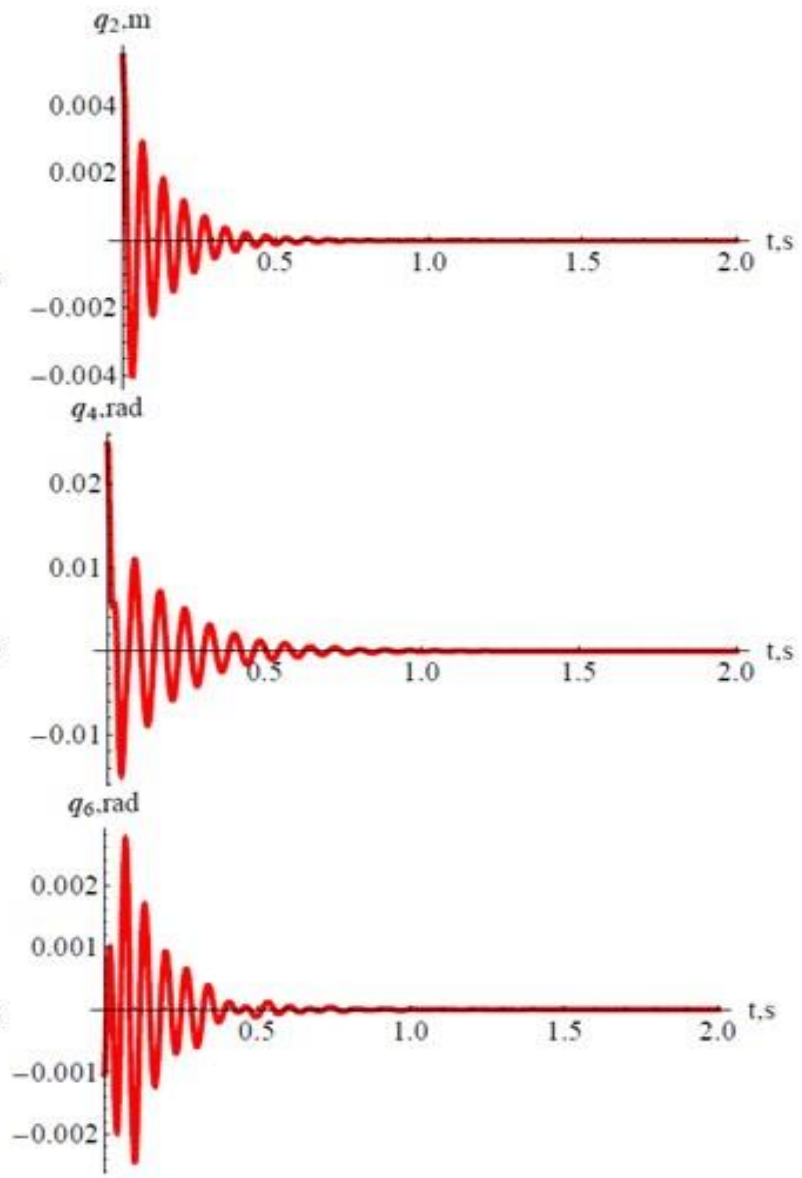


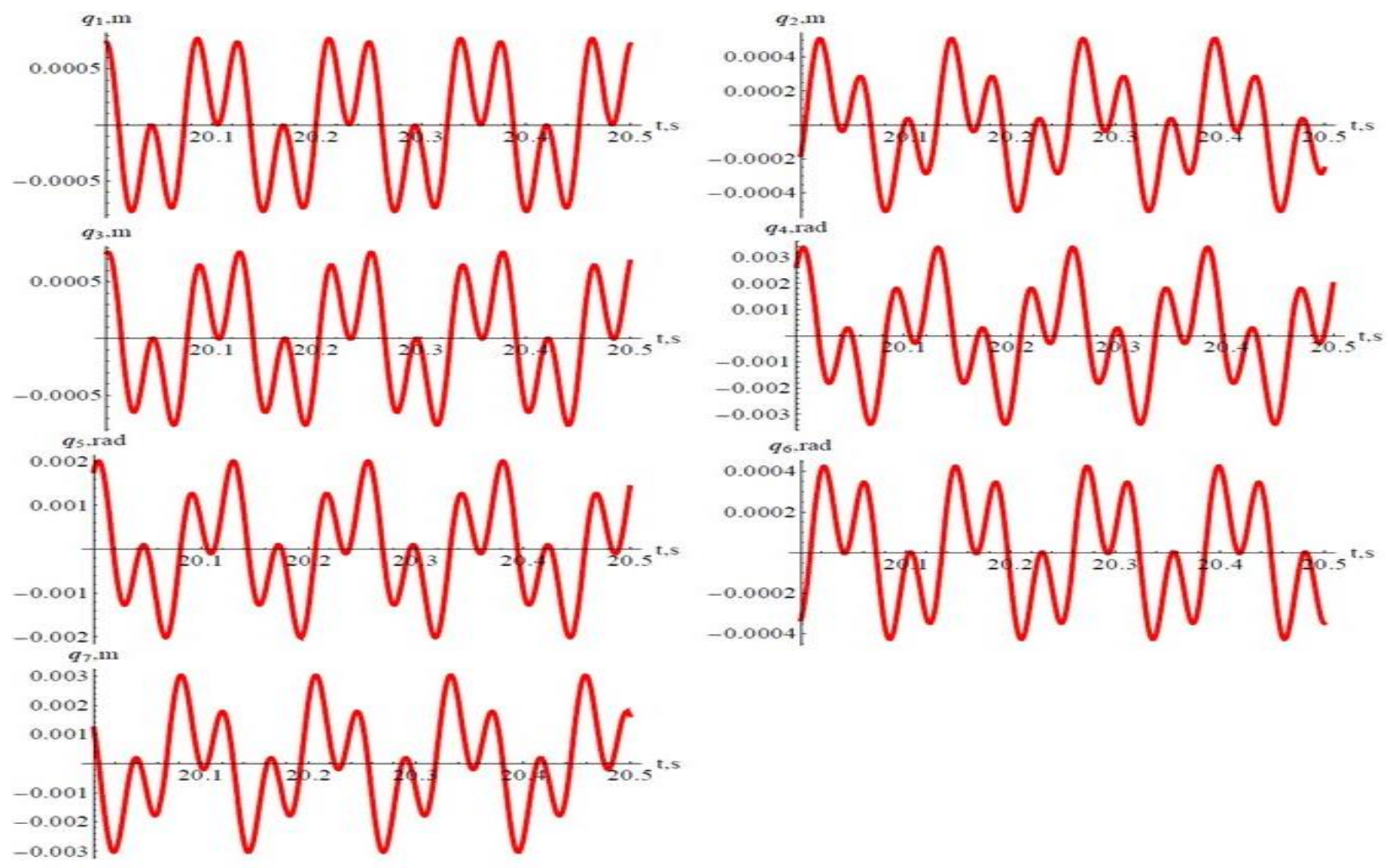

Fig. 6 Diagrams of forced vibrations of the system at all generalized coordinates
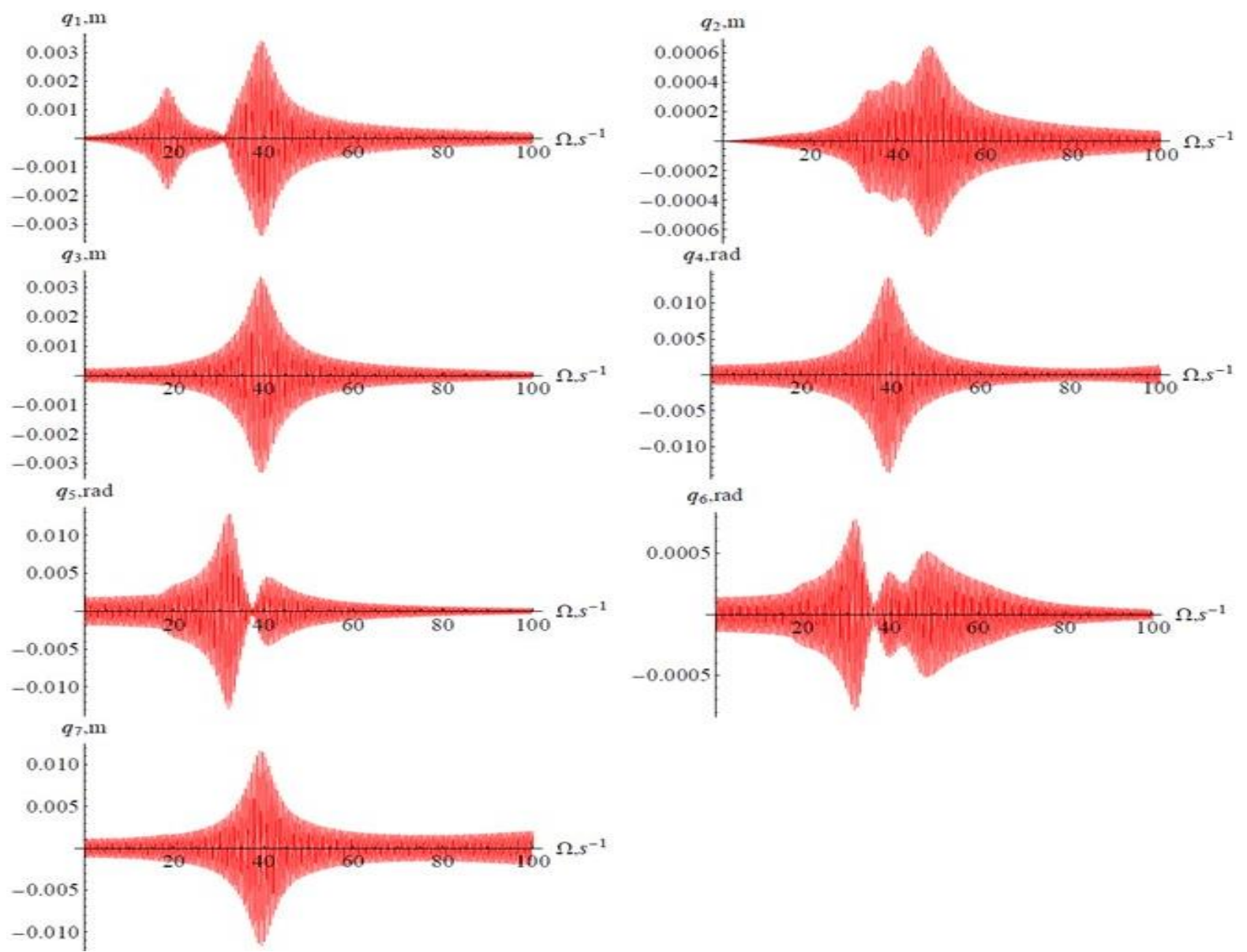

Fig. 7 Diagrams of amplitude-frequency characteristics of the system at all generalized coordinates

The obtained results show that the study design of the small model of fertilizer hopper will work outside the resonance frequencies $(7.96 \mathrm{~Hz})$. The properties of the elastic-damping elements are selected so that free vibrations are damped within 1 s. Figs. 5, 6 and 7 depict the major achievement of this work. These graphs show that free oscillations decrease over a limited period of time, which is about $1 \mathrm{~s}$. This really facilitates the avoiding of resonant zones. Forced 
uniform oscillations are reproduced in time, and do not experience the influence of free oscillations. Such a result falls within the set goal. These computational results are in tune with the results of modelling that showed a scaled model not entering into resonance at any frequency from the broad range of frequencies of the electromagnetic actuator. In order to improve the operation of the hopper elasticity coefficients of elastic elements may be changed [16]. Thus resonant zones of operation are avoided should any change in operating frequency of the electromagnet become necessary.

\section{Conclusion}

A methodology for dynamic analysis of the hopper of a fertilizer applying machine is proposed.

After the design development of the hopper, specific values for the masses, mass moments of inertia, geometry, elastic and damping characteristics of elastic-damping elements exciting forces will be received.

With these input data using the developed methodology numerical and graphical results for forced vibrations of the device can be obtained. These will provide for the optimization of the design in order to avoid resonance of the system.

Thus, the wear of the apparatus will decrease while improving the quality of his work. The results of this article can be used to further improve the quality of the point of fertilization in the application of forced vibration of the hopper car.

\section{References}

1. Song, Z. 2015. Design and test of teamwork machine rototilling and fertilizing in paddy field, Journal of Agricultural Mechanization Research 7: 147-150.

2. Hanna, M. 2016. Estimating the field capacity of farm machines, Agriculture and Natural Resources. Available at:

http://lib.dr.iastate.edu/cgi/viewcontent.cgi?arti-

cle $=1003 \&$ context=pubs_agdm.

Last assessed: 10.05.2018.

3. Li, Y. R.; Yang, L.T. 2015. Sugarcane agriculture and sugar industry in China, Sugar Tech 17(1): 1-8. http://dx.doi.org/10.1007/s12355-014-0342-1.

4. Patent 19007 RK. 2008. Bulk material distributing device.

5. Matveev, Y.; Valieva, E.N.; Trubetskaya, O.V.; Kislov, A.G. 2016. Globalization and regionalization: Institution Aspect, IEJME-Mathematics Education 11(8): 3114-3126.

6. Kaplan, J. 2004. Fertilizer applying quality. Minneapolis.

7. Cakmak, B.; Aykas, E.; Onal, I.; Cakir, E. 2010. The performance of developed rotary tiller fitted with pneumatic seeder, Bulgarian Journal of Agricultural Science 16(6): 801-810 (2010).

8. Schuman, A.W. 2010. Precise placement and variable rate fertilizer application technologies for horticultural crops. Journal Hort Technology 20(1): 34-40.

9. Schumann, A.W.; Hostler, K.H. 2010. Variable rate application technology for enhanced production efficiency. Paran: Paran University of Agronomy.
10. Zuev, F.G. 1976. Pneumatic transport of cereal processing plants. Moscow: Kolos.

11. Biocca, M. 2016. Evaluating a filtering and recirculating system to reduce dust drift in simulated sowing of dressed seed and abraded dust particle characteristics. Pest Management Science. https://doi.org/10.1002/ps.4428.

12. Foqué, D., et al. 2014. Characterisation of different pneumatic sowing machines, Aspects of Applied Biology 122: 77-84.

13. Manzone, M., et al. 2015. Potential external contamination of pneumatic seed drills during sowing of dressed maize seeds.Pest management science. https://doi.org/10.1016/j.cropro.2015.06.002.

14. Angelov, I.; Slavov, V. 2006. Matrices mechanics. Kinematics. Sofia: Avangard Prima. https://doi.org/10.1007/s11012-013-9823-7.

15. Tsirkunov, Y.M.; Panfilov, S.V. 1994. Dusty gas flow around bodies: effects of non-elastic reflection of particles. In Proceeding of the 2nd European Fluid Mechanics Conference, September 20-24, Warsaw, p. 274.

16. Eskhozhin, D.; Nukeshev, S.; Akhmetov, E.; Zhaksylykova, Z.; Balabekova, A. 2013. Technical problems of intra-soil applying of mineral fertilizers and their solution, In: Proceedings of the Innovation Convent Kuzbass: Education, Sciences, Innovations, Kemerovo, Russia.

S. Nukeshev, V. Slavov, D. Karaivanov, A. Balabekova, Z. Zhaksylykova

\section{FORCED VIBRATIONS OF THE HOPPER OF FERTILIZER APPLYING MACHINE}

S u m m a r y

The cultivation of crops is not only an important part of the economic sector of the state, but also the condition of food security of citizens. A key method of increasing yields is fertilizer in the soil. However, most machines cannot efficiently allocate and apply the fertilizer in the soil. In this regard, a dynamic model of the hopper of fertilizer applying machine is created. A methodology for determination of the natural frequencies of the mechanic system is proposed. Using matrix mechanics, equations for determining the kinetic and potential energy are derived. In the paper differential equations for description of forced small-amplitude vibrations of the system are composed and solved. Thanks to them the natural frequencies of the system are determined. The results are intended for research and optimization of a real machine, developed in KazATU - Astana, Republic of Kazakhstan.

Keywords: dosing device, dynamic model, fertilizing machine, vibrations, mechanical system.

Received March 09, 2017

Accepted December 12, 2018 